\title{
Monte-Carlo Study Based on Real Coordinates for Perpendicularly Injected High-Energy Ions in the LHD High-Beta Plasma
}

\author{
Ryosuke SEKI ${ }^{\mathrm{a})}$, Yutaka MATSUMOTO, Yasuhiro SUZUKI ${ }^{1)}$, Kiyomasa WATANABE ${ }^{1)}$, \\ Kiyotaka HAMAMATSU ${ }^{2)}$ and Masafumi ITAGAKI \\ Graduate School of Engineering, Hokkaido University, Sapporo 060-8628, Japan \\ ${ }^{1)}$ National Institute for Fusion Science, Toki 509-5292, Japan \\ 2) Japan Atomic Energy Agency, Naka 311-0193, Japan
}

(Received 4 November 2009 / Accepted 28 December 2009)

\begin{abstract}
We present a study of ions produced by the perpendicular neutral beam in the high-beta plasma of Large Helical Device (LHD) that uses the Monte-Carlo code based on orbit following in real coordinates with Coulomb collisions. For no neutrals outside the plasma, the results indicate that there are remarkable differences in the distribution functions that depend on whether the loss boundary is set on the vacuum vessel wall or on the last closed flux surface (LCFS). Also, for finite neutral density $n_{\mathrm{H}}=10^{18} \mathrm{~m}^{-3}$, we find that the minor radial profile of the distribution function slightly differs from that for the infinite neutral density case, which corresponds to the loss boundary being set at the LCFS.
\end{abstract}

(C) 2010 The Japan Society of Plasma Science and Nuclear Fusion Research

Keywords: re-entering particle, LHD, perpendicular neutral beam, charge exchange loss, Monte-Carlo simulation

DOI: $10.1585 /$ pfr.5.014

A perpendicular neutral beam (NB) injector and three tangential-NB injectors haves been installed on Large Helical Device (LHD). Using these NBs, the volume-averaged beta $\langle\beta\rangle$ has reached $5 \%$ in recent LHD experiments [1]. In the high-beta plasma of LHD, it has been pointed out that the beam pressure and/or the pressure anisotropy significantly affect the properties of magneto-hydrodynamics equilibrium and stability [2]. Therefore, it is important to identify the contribution of the beam-pressure to the total plasma pressure. Since the beam pressure is calculated from the distribution function of high-energy ions produced by the NBs, it is also important to investigate the distribution function.

To analyze the distribution function of the highenergy ions in LHD, we developed a Monte-Carlo code (MORH) [3] based on an orbit following in real coordinates with Coulomb collisions [4]. In this code, we set the particle-loss boundary on the vacuum vessel (VV) wall instead of the last closed flux sufrace (LCFS), because we can follow the ion orbit until the VV wall. Thus, reentering particles [5,6], which repeatedly pass into and out of the LCFS, can be treated. Additionally, the effect of charge exchange (CX) loss [7] on the re-entering particles can be investigated. This code has already been applied to the ions produced by the tangential-NBs in LHD, and it was shown that the re-entering particles play an important role in the analyzing the distribution function of ions

\footnotetext{
author's e-mail: seki.ryohsuke@lhd.nifs.ac.jp

a) present address: National Institute for Fusion Science, Toki 509-5292, Japan
}

produced by the tangential-NBs for $B_{\mathrm{ax}}=3 \mathrm{~T}$ [3]. On the other hand, it has also been shown that most of the protons produced by the perpendicular-NB become the re-entering particles in high-beta plasmas [8]. Thus, the re-entering particles may play a more important role in the distribution function in the high-beta plasma.

In this paper, we apply the MORH code to the protons produced by the perpendicular-NB in the high-beta plasma of LHD in the case of $B_{\mathrm{ax}}=3 \mathrm{~T}$ for comparison with the results in Ref. [3]. For the calculation, we assume that the NB (40 keV, $1 \mathrm{MW}$ ) is injected torus-outside at the horizontally elongated poloidal cross-section in the equatorial plane. The number of the high-energy protons produced by the NB is assumed to be uniform on the beam line, and their initial energy $E_{0}$ is only $40 \mathrm{keV}$. Other energy components $\left(E_{0} / 2, E_{0} / 3\right)$ of the high-energy protons are ignored in the present study. The high-beta equilibrium magnetic field $\left(\langle\beta\rangle=2.7 \%, B_{\mathrm{ax}}=3 \mathrm{~T}\right)$ is calculated using the HINT code [9]. This is exactly same as that shown Fig. 1 (b) of Ref. [8], in which the magnetic axis and the center of the LCFS are at $\simeq 3.90 \mathrm{~m}$ and $\simeq 3.71 \mathrm{~m}$, respectively. For simplicity, it is assumed that the temperature and density of the background plasma are constant throughout the region inside the VV wall. The background plasma density is $10^{20} \mathrm{~m}^{-3}$ and the ion and electron temperature is $1.0 \mathrm{keV}$.

Figure 1 shows the distribution functions calculated by the MORH code. Here, Figs. 1 (a), (b) and (c) show the distribution function $F(\rho)$ in the minor radius $(\rho)$ space, distribution function $F(v)$ in the velocity $(v)$ space, and distribution function normalized by its maximum value in 


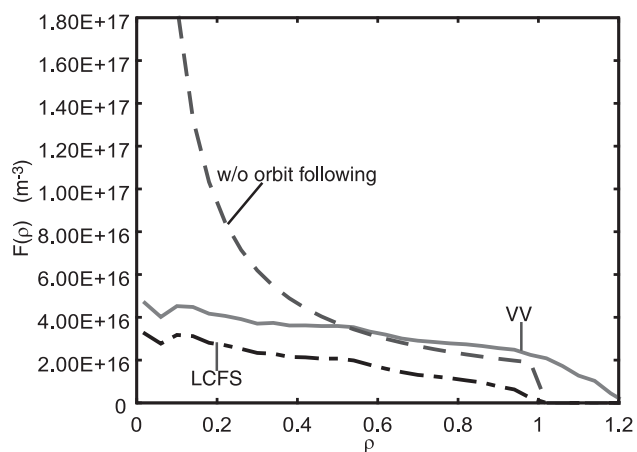

(a) rho space

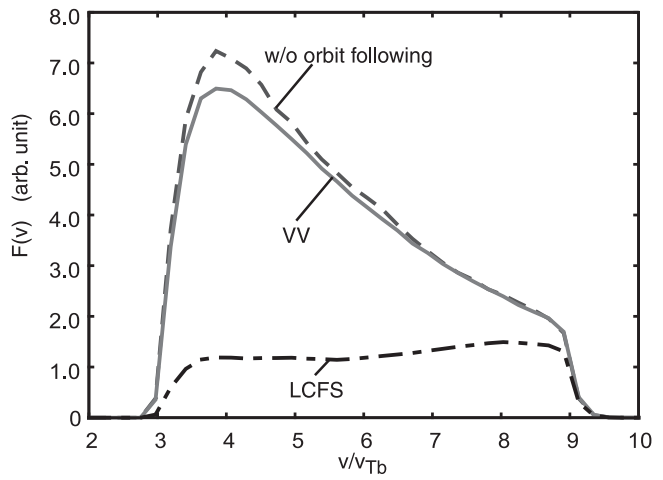

(b) v space

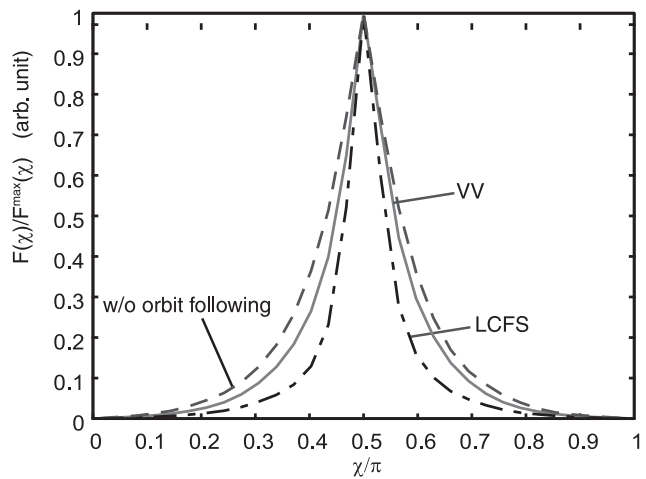

(c) pitch angle space

Fig. 1 Various distribution functions calculated by the MORH code. In Fig(b), the horizontal axis is normalized by the background-ion thermal velocity.

the pitch angle $(\chi)$ space, $F(\chi) / F^{\max }(\chi)$, respectively. In Fig. 1, the solid lines (labeled VV) denote the distribution functions when the loss boundary is set on the VV wall, and dashed-dotted lines (labeled LCFS) denote the distribution functions when the loss boundary is set on the LCFS, which corresponds to the conventional analysis model using the Boozer coordinates. The distribution functions without orbit following are shown by dashed lines.

In Fig. 1 (a), there are remarkable differences between $F^{\mathrm{VV}}(\rho)$ and $F(\rho)$ without orbit following. The ions produced by the perpendicular-NB tend to be helically trapped banana-orbit particles in LHD [8]. In the high beta plasma of LHD, the banana-orbit particle moves across the flux surfaces, and it deviates significantly from the flux surface on which its initial point is set. Therefore, the profile of
$F(\rho)$ with orbit following becomes broad. It can also be seen from Fig. 1 (a) that $F^{\mathrm{LCFS}}(\rho)$ is smaller than $F^{\mathrm{VV}}(\rho)$ over the entire region of $\rho$. This result shows that there are many re-entering particles independent of $\rho$ under these conditions. In particular for $\rho>1, F^{\mathrm{VV}}(\rho)>10^{16} \mathrm{~m}^{-3}$, while $F^{\mathrm{LCFS}}(\rho)=0$. This difference is caused by the loss boundary condition. When the loss boundary is set on the LCFS, the re-entering particles are regarded as lost particles. Therefore, $F^{\mathrm{LCFS}}(\rho)$ does not exist for $\rho>1$.

In Fig. 1 (b), the reason $F(v)=0$ for $v<3$ is that in this code, the ion orbits are followed until the ions are lost or thermalized. In this coe, a thermalized particle is defined as an ion for which the velocity is less than three times the background-ion thermal velocity. We note that the average orbit following time is about $6.5 \mathrm{~ms}$ in the present calculation. $\quad F^{\mathrm{VV}}(v)$ is less than $F(v)$ without orbit following (Fig. 1 (b)). This difference comes from the lost particles on the VV wall, which is a result of orbit following. As seen in Fig. $1(\mathrm{~b}), F^{\mathrm{LCFS}}(v)$ is much smaller than $F^{\mathrm{VV}}(v)$, and that the shape of $F^{\mathrm{LCFS}}(v)$ is flat. In the high-beta plasma of LHD, most ions produced by the perpendicularNB are the re-entering particles. There is a possibility that $F^{\mathrm{LCFS}}(v)$ is underestimated when the loss boundary is set on the LCFS, because the re-entering particles are regarded as the lost particles.

In Fig. 1 (c), the normalized $F(\chi)$ profile is localized at $\chi \simeq 0.5 \pi$, which corresponds to $\chi$ at the starting point. The reason is that the estimated energy relaxation time and deflection time are about 5.3 and $22 \mathrm{~ms}$, respectively, and the averaged orbit following time is close to $5.3 \mathrm{~ms}$. In Fig. $1(\mathrm{c})$, the normalized $F^{\mathrm{LCFS}}(\chi)$ is smaller than the normalized $F^{\mathrm{VV}}(\chi)$ near $0.4 \pi$ and $0.6 \pi$. The ions with $\chi \simeq 0.5 \pi$ are the banana-orbit particles. When $\chi$ of ions change from $\simeq 0.5 \pi$ to $\simeq 0.4 \pi$ or $0.6 \pi$ because of the collisions, the ions become chaotic-orbit particles. For the conditions used, most chaotic-orbit particles also become the re-entering particles [8]. Therefore, $F^{\mathrm{LCFS}}(\chi)$ is underestimated near $0.4 \pi$ or $0.6 \pi$.

We also investigate the effect of CX loss on the reentering particles. In this paper, we assume that the CX loss occurs only with the hydrogen atoms. The density of the hydrogen atoms $\left(n_{\mathrm{H}}\right)$ is assumed only outside the LCFS. Figure 2 shows the effect of CX loss on $F(\rho)$. Note that $F(\rho)$ for the $n_{\mathrm{H}}=0$ corresponds to $F^{\mathrm{VV}}(\rho)$ shown in Fig. $1(\mathrm{a})$, and that $F(\rho)$ for $n_{\mathrm{H}}=\infty$ represents $F^{\mathrm{LCFS}}(\rho)$ in Fig. 1 (a). $n_{\mathrm{H}}=10^{18} \mathrm{~m}^{-3}$ is estimated by the degree of vacuum obtained in the LHD experiments. In Fig. 2, $F(\rho)$ decreases with increasing density of the hydrogen atoms. However, as the density of the hydrogen atoms increases, the shape of $F(\rho)$ slightly changes for $\rho<1$. For the $n_{\mathrm{H}}=10^{18} \mathrm{~m}^{-3}, F(\rho)$ is almost the same as $F^{\mathrm{LCFS}}(\rho)$, which implies that the path of the re-entering particle outside the LCFS is much longer than the mean free path of the CX reaction.

We investigated the distribution function of the ions produced by the perpendicular-NB in the high beta plasma 


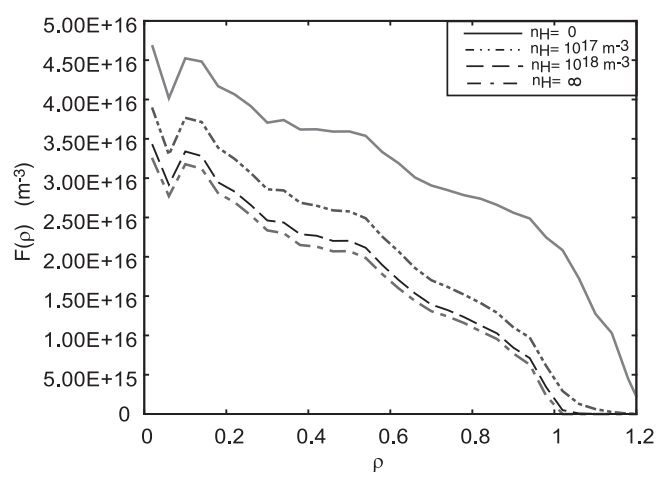

Fig. 2 Effect of charge exchange loss on $F(\rho)$.

of LHD using the MORH code. For no neutrals outside the plasma, there are remarkable differences in the distribution functions that depend on whether the loss boundary is set on the VV wall or on the LCFS. Also, the shape of $F(\rho)$ for the $n_{\mathrm{H}}=10^{18} \mathrm{~m}^{-3}$ slightly differs from that for $n_{\mathrm{H}}=\infty$. In the analysis of alpha particles, the mean free path of the CX reaction lengthens because of high-energy of the alpha particles. In this analyses, the effect of the re-entering particles on the distribution function may be more important. In the high-beta experiments of LHD, the typical operation magnetic field strength is low $\left(B_{\mathrm{ax}} \simeq 0.5 \mathrm{~T}\right)$; hence, in future investigations, we will study the distribution function of protons produced by NBs at these low magnetic field strengths. It is also an important issue to study the heating efficiency by the NBs; hence, the heating efficiency of the NBs will be analyzed using the MORH code in the near future.

We thank Prof. S. Oikawa (Hokkaido University) for fruitful discussions and Dr. M. Osakabe (NIFS) for providing the information on the LHD NB injector systems. This work was supported by the collaborative research program of NIFS (NIFS08KLHH309) and was partially supported by a Grant-in-Aid for the Scientific Research (B) (18360445) of the Japan Society for the Promotion of Science (JSPS).

[1] K.Y. Watanave et al., in Proc. of ITC-17 and ISWS-16 Toki I-13 (2007).

[2] W.A. Cooper et al., Fusion Sci. Technol. 50, 245 (2006).

[3] R. Seki et al., submitted to Plasma Fusion Res. (2009).

[4] K. Hamamatsu et al., Plasma Phys. Control. Fusion 49, 1955 (2007).

[5] R.H. Fowler et al., Nucl. Fusion 30, 997 (1990).

[6] K. Hanatani and F.-P. Penningsfeld, Nucl. Fusion 32, 1769 (1992).

[7] R.J. Goldston et al., J. Comput. Phys. 46, 61 (1981).

[8] R. Seki et al., Plasma Fusion Res. 3, 016 (2008).

[9] K. Harafuji et al., J. Comput. Phys. 81, 169 (1989). 